\title{
Establishment and genomic characterizations of patient-derived esophageal squamous cell carcinoma xenograft models using biopsies for treatment optimization
}

Jianling Zou ${ }^{1 \dagger}$, Ying Liư ${ }^{2 \dagger}$, Jingyuan Wang ${ }^{1}$, Zhentao Liu', Zhihao Lu', Zuhua Chen ${ }^{1}$, Zhongwu Li ${ }^{3}$ Bin Dong ${ }^{3}$, Wenwen Huang ${ }^{1}$, Yanyan $\mathrm{Li}^{1}$, Jing Gao ${ }^{1^{*}}$ and Lin Shen ${ }^{1^{*}}$ (i)

\begin{abstract}
Background: Squamous cell carcinoma is the dominant type of esophageal cancer in China with many patients initially diagnosed at advanced stage. Patient-derived xenografts (PDX) models have been developed to be an important platform for preclinical research. This study aims to establish and characterize PDX models using biopsy tissue from advanced esophageal cancer patients to lay the foundation of preclinical application.

Methods: Fresh endoscopic biopsy tissues were harvested from patients with advanced esophageal cancer and implanted subcutaneously into NOD/SCID mice. Then, the PDXs were serially passaged for up to four generations. Transplantation was analyzed and genomic characteristics of xenografts were profiled using next-generation sequencing.

Results: Twenty-five PDX models were established (13.3\%, 25/188). The latency period was $75.12 \pm 19.87$ days (50-120 days) for the first passage and it decreased with increasing passaging. Other than tumor stages, no differences were found between transplantations of xenografts and patient characteristics, irrespective of chemotherapy. Histopathological features and chemosensitivity of PDXs were in great accordance with primary patient tumors. Each PDX was assessed for molecular characteristics including copy number variations, somatic mutations, and signaling pathway abnormalities and these were similar to patient results.
\end{abstract}

Conclusions: Our PDX models were established from real time biopsies and molecularly profiled. They might be promising for drug development and individualized therapy.

Keywords: Patient-derived xenograft model, Esophageal squamous cell carcinoma, Biopsy, Genomic characterizations

\section{Background}

Esophageal cancer is the third most common cancer and the fourth leading cause of cancer death in all cancer types in China [1]. Esophageal squamous cell carcinoma

\footnotetext{
*Correspondence: gaojing_pumc@163.com; linshenpku@163.com †Jianling Zou and Ying Liu contributed equally to this work 1 Department of Gastrointestinal Oncology, Key Laboratory of Carcinogenesis and Translational Research (Ministry of Education), Peking University Cancer Hospital \& Institute, Fu-Cheng Road 52, Hai-Dian District, Beijing 100142, China

Full list of author information is available at the end of the article
}

(ESCC) is the predominant histological type, representing more than $95 \%$ of all cases in China $[2,3]$. Most patients are initially diagnosed at advanced stages and cannot be surgically removed. At this time, chemotherapy and radiotherapy are primary approaches for treatment but this offers little benefit: five-year survival has not changed in decades $[4,5]$. Due to the limited therapeutic agent available and frequent drug resistance, it is urgent to exploit new agents and explore the mechanisms of drug resistance [6]. 
Recent studies indicate that various tumorigenic signaling pathways are involved in ESCC, such as tumor growth, cell cycle, angiogenesis, invasion and apoptosis [7-9], so targeting these signaling pathways may be strategies for treating ESCC. Target therapy also showed exciting effect when combined with immunotherapy [10-12]. However, there is no specific agent for targeted therapy in ESCC. Therefore it is urgent to identify promising targets for therapy to improve the survival of ESCC patients.

Recently, patient-derived xenograft (PDX) models have been developed for translating basic research into clinical solutions and these offer advantages over cell line-based model $[13,14]$. Because PDX involves transplanting cancer patients tissue directly into immunocompromised mice, genetic information, immunohistological markers and chemosensitivity are correlative to the patient and can be applied to evaluate new antitumor drugs $[6,13$, $15,16]$. Thus PDX models provide an irreplaceable platform to study biological and genetic alterations, as well as potential anticancer therapies.

Little literature exists to describe PDX models of ESCC, and most were established using surgical tissues with relatively earlier tumor stages [17-21]. However, patients with advanced staged tumors are better for evaluating the efficacy of new agents, and real-time endoscopic biopsy is the primary way to obtain tumor tissues in daily clinical works. Thus, PDX models generated with endoscopic biopsies from advanced patients may be more useful for drug development and guiding individualized therapy.

In this study, we established PDX models of ESCC with endoscopic biopsy tissues successfully and assessed the clinical and pathological factors associated with engraftment as well as the chemosensitivity of xenograft. Finally, genomic characterizations of PDXs were identified to explore new agents of targeted therapy in ESCC.

\section{Methods}

\section{Patients and tissue samples}

The research proposal had been approved by the Medical Ethics Committee of Peking University Cancer Hospital according to principles of the Declaration of Helsinki. Written informed consent was obtained from all study participants for their information to be stored in the hospital database and used for future research at the time of follow-up ascertainment. All the patients with pathologically confirmed esophageal carcinoma and available endoscopic biopsied samples were included in the study.

\section{Establishment of PDX models}

NOD/SCID mice (6 weeks) were from Beijing Vital River Laboratory Animal Technology Co., Ltd. Two tissue fragments $\left(\sim 2 \times 2 \times 2 \mathrm{~mm}^{3} /\right.$ fragment $)$ from fresh biopsies were obtained from each patient ( $\mathrm{P} 0=$ passage zero), and were subcutaneously implanted into the flank of each mouse under sterile conditions. Tumor growth was assessed by palpation or Vernier calipers twice weekly. The established PDX model was passage 1 (P1). Mice were euthanized and harvested fresh tumor fragments were re-implanted into other mice when P1 tumors reached $\sim 750 \mathrm{~mm}^{3}$. If animals showed disease, the tumor was collected when palpable. Subsequent passages were P2, P3, and P4. All procedures were performed under sterile conditions at Peking University Cancer Hospital specified-pathogens free facility and carried out in accordance with the Guide for the Care and Use of Laboratory Animals of the NIH. Each model derived from individual patients was passaged for up to four generations. At each passage, tumor tissues were cryopreserved (90\% FBS and 10\% DMSO), snap-frozen in liquid nitrogen for future use, and fixed in neutral buffered formalin for histological examination.

\section{RNA extraction and quantitative real-time PCR}

Total RNA was extracted from fresh PDX samples with TRIzol reagent (Invitrogen) in accordance with the manufacturer's protocol. RNA concentration was quantified using a Nanodrop (Thermo Scientific, Hemel Hempstead, UK) and diluted to $100 \mathrm{ng} / \mathrm{ml}$ in RNase-free water before using the TransScript II One-Step gDNA Removal and cDNA Synthesis SuperMix kit (TransGen Biotech) according to the introduction. The cDNA was kept at $-20{ }^{\circ} \mathrm{C}$ until used for qPCR. Quantitative real-time RTPCR analyses were performed using the $\mathrm{SYBR}^{\circledR}$ Green Realtime PCR Master Mix (TOYOBO, BIOTECH CO., LTD), the BIO-RAD CFX96TM Real-Time System and Bio-Rad CFX Manager 2.1 software (Bio-Rad Laboratories, Inc., USA). The primer sequences for human FGF3 were: forward, 5'-ATGCTTCGGAGCACTACAGC-3', and reverse, 5'-CCGTTCACAGACACGTACCA-3'. The primer sequences for human FGF4 were: forward, 5'-CTATGGCTCGCCCTTCTTCA-3', and reverse, 5'-CCATTCTTGCTCAGGGCGAT-3'. The primer sequences for human FGF19 were: forward, 5'-AGATCAAGGCAGTCGCTCTG-3', and reverse, 5'-GAGTACTGAAGCAGCCCCTG-3'. The primer sequences for human GAPDH were: forward, 5'-TTTGGTATCGTGGAAGGACT-3', and reverse, 5' -AGTAGAGGCAGGGATGATGT-3'. The reaction conditions were an initial step of $95^{\circ} \mathrm{C}$ for $60 \mathrm{~s}$, followed by 40 cycles of denaturation at $95^{\circ} \mathrm{C}$ for $15 \mathrm{~s}$ and annealing for $15 \mathrm{~s}$, and extension at $72{ }^{\circ} \mathrm{C}$ for $45 \mathrm{~s}$. Expression was normalized for RNA loading using GAPDH primers designed to span an intron and relative expression in each sample was calculated. To compare the difference of mRNA expression, fold changes were calculated by dividing the samples into 
two groups: $\mathrm{CNV} \geq 5$ or $\mathrm{CNV}<5$. Results are expressed as means \pm SEMs. The semi-quantitative RT-PCR analyses from one individual experiment were repeated three times with comparable results.

\section{H\&E staining}

Histopathology of primary P0 tumors and xenografts were evaluated using $H \& E$ staining according to standard method with an H\&E staining kit (C0105, Beyotime, China). Results were reviewed by two independent pathologists.

\section{Chemosensitivity of PDX model}

Patient-derived xenografts models with 3 or more passages were used to evaluate chemosensitivity. Tumor bearing mice $\left(\sim 150 \mathrm{~mm}^{3}\right.$ tumors) were randomized into two groups: treatment (paclitaxel, $10 \mathrm{mg} / \mathrm{kg}$; platinum, $5 \mathrm{mg} /$ $\mathrm{kg}$, ip) and controls (saline). Treatment regimens were consistent with patient treatment and doses were determined from the literature [22-24]. Treatment was given once weekly for 3 cycles. Tumor size was measured twice weekly as described and tumor volume $(\mathrm{V})$ was calculated as follows: $\mathrm{V}=\mathrm{L} * \mathrm{~W}^{2} / 2$ (L, length, long diameter of tumor; W, width, short diameter of tumor). All animal procedures were approved by the ethics committee for animal experiments at Peking University Cancer Hospital. Tumor growth inhibition $(\mathrm{TGI})=(1-\Delta \mathrm{T} / \Delta \mathrm{C}) \times 100 \%$ $(\Delta \mathrm{T}=$ tumor volume change of the drug-treated group on the final day of the study, $\Delta \mathrm{C}=$ tumor volume change of the control group on the final day of the study).

\section{Targeted next-generation sequencing and data analysis} Genomic DNA was extracted from P4 xenografts of each PDX model using a QIAamp DNA Mini Kit (QIAGEN Ltd., Crawley, UK) according to the manufacturer's instructions. Extracted DNA was evaluated using a Qubit fluorometer (Invitrogen, Carlsbad, CA) and 1\% agarose gel electrophoresis. A custom 483 cancer-related gene panel was used (Additional file 1: Table S1) [25]. The capture-based library was generated from $500 \mathrm{ng}$ of DNA from each sample using a KAPA Hyper Prep Kit according the manufacture's instruction (Kapa Biosystems, Boston, MA, USA), followed by Agilent's SureSelectXT Target Enrichment System (Agilent Technologies, Santa Clara, CA). Library quality was assessed using Agilent 2100 Bioanalyzed on-chip electrophoresis (Agilent Technologies, Inc), and the library was quantified with an Agilent QPCR NGS Library Quantification Kit (Agilent Technologies, Inc). The library was sequenced on an Illumina Hiseq 2000 system (Illumina, San Diego, CA).

Sequencing adapters and low quality reads were filtered and the final Q20 and Q30 of all samples were > 90 and $>85 \%$, respectively. The BWA software with default parameters was used to align sequencing reads to the human reference hg19 genome, and Picard was used to mark duplications. The aligned reads achieved coverage of $>99 \%$ of the target region with a mapping rate of $>95 \%$. The average sequencing depth was more than $1200 \times$ per sample. Mutations were called using Samtools, Mutect, and Varscan software, and were annotated using Annovar software. CNV analysis was performed using EventWise Testing algorithm according to previous reports [26]. A neutral copy number for each exon for each gene was established using normal lymphocyte samples.

\section{Statistical analysis}

Data were assessed for clinicopathological characteristics and transplantation success using a Chi squared test. An unpaired two-tailed Student's $t$ test was used to analyze the latency period of xenografts. Tumor growth between groups was compared using repeated-measured analysis of variance. A $p$ value $<0.05$ was considered statistically significant. Statistical analysis was performed with SPSS software for Windows, version 21 (SPSS Inc., Chicago, IL, USA).

\section{Results \\ Establishment of PDX models and patient clinical characteristics}

A total of 188 esophageal carcinoma samples were obtained by endoscopic biopsy, and 40 PDX models were established in NOD/SCID mice at P1 (Fig. 1a; transplantation success 21.3\%) Along with serial passaging, the transplantation rates from P1 to P2, P2 to P3, P3 to P4 were $70 \%(28 / 40), 89.3 \%(25 / 28), 100 \%$ $(25 / 25)$, respectively. After the fourth generation, the PDX models became stable without further change in model formation, which could be used in future study. The overall transplantation rate for PDX models in our study was $13.3 \%(25 / 188)$. The median latency period of xenografts was decreased along with serial passage with $75.12 \pm 19.87$ days, $56.96 \pm 17.17$ days, $45.88 \pm 21.38$ days, and $28.04 \pm 16.23$ days at P1, P2, P3, and $\mathrm{P} 4$ passage (Fig. 1b, $p<0.05$ ). Due to tissue limitations for the first two passages, a bio-specimen bank of xenografts from passage three were established, including formalin fixed paraffin-embedded blocks, viably cryopreserved and snap frozen tissues. Cryopreserved xenografts could be re-grown in mice, providing a renewable tissue source for future usage.

No difference was observed between transplantation rates and patient characteristics except for tumor stage (Additional file 1: Table S2). Transplantation rates of biopsied samples from stage III or IV $(17.7 \%, 22 / 124)$ was much higher than that from early stages $(0,0 / 19$, $p<0.05$ ). Table 1 shows data for the 25 PDX models established. All tissues were from males and from 

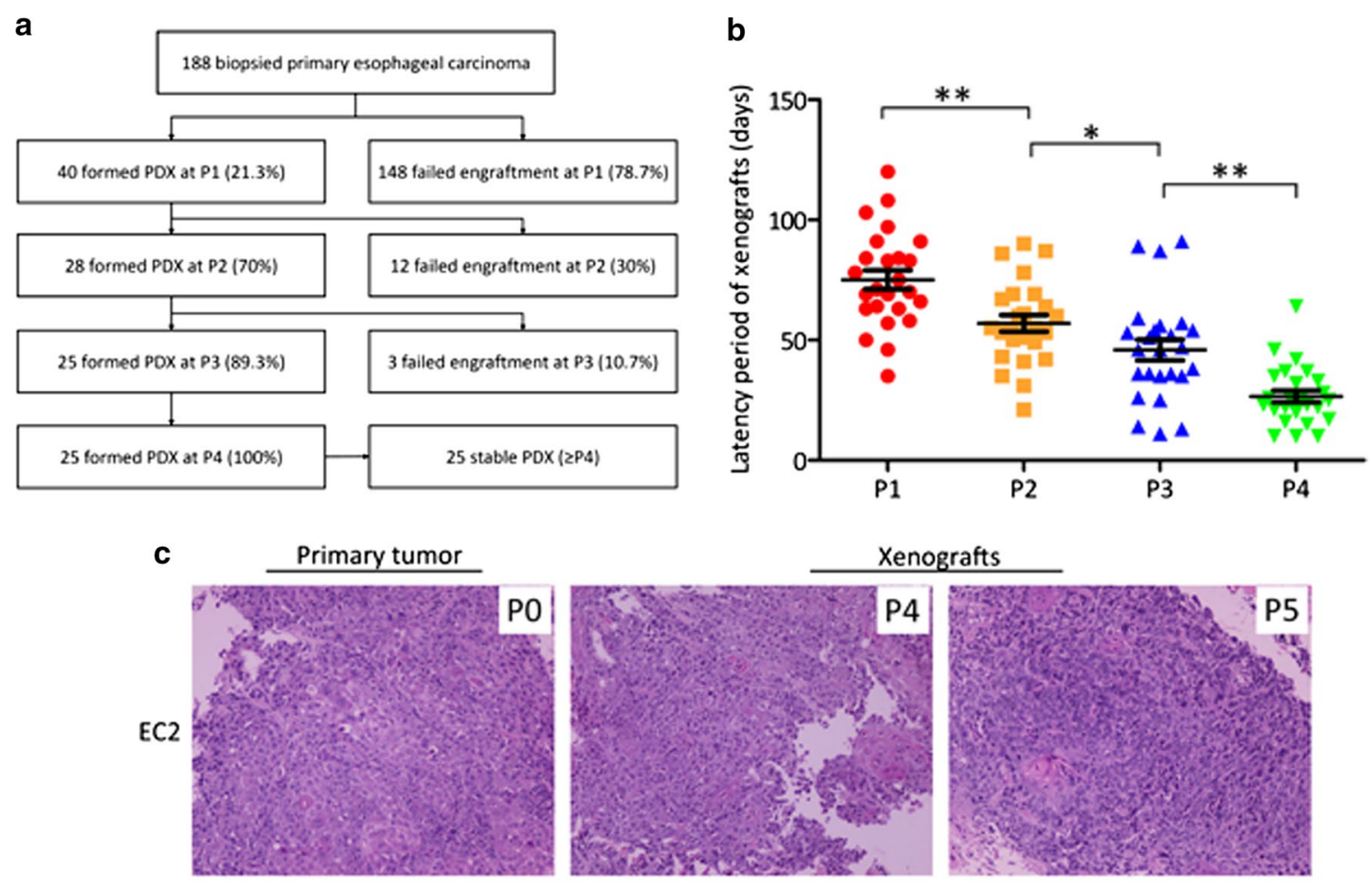

Xenografts
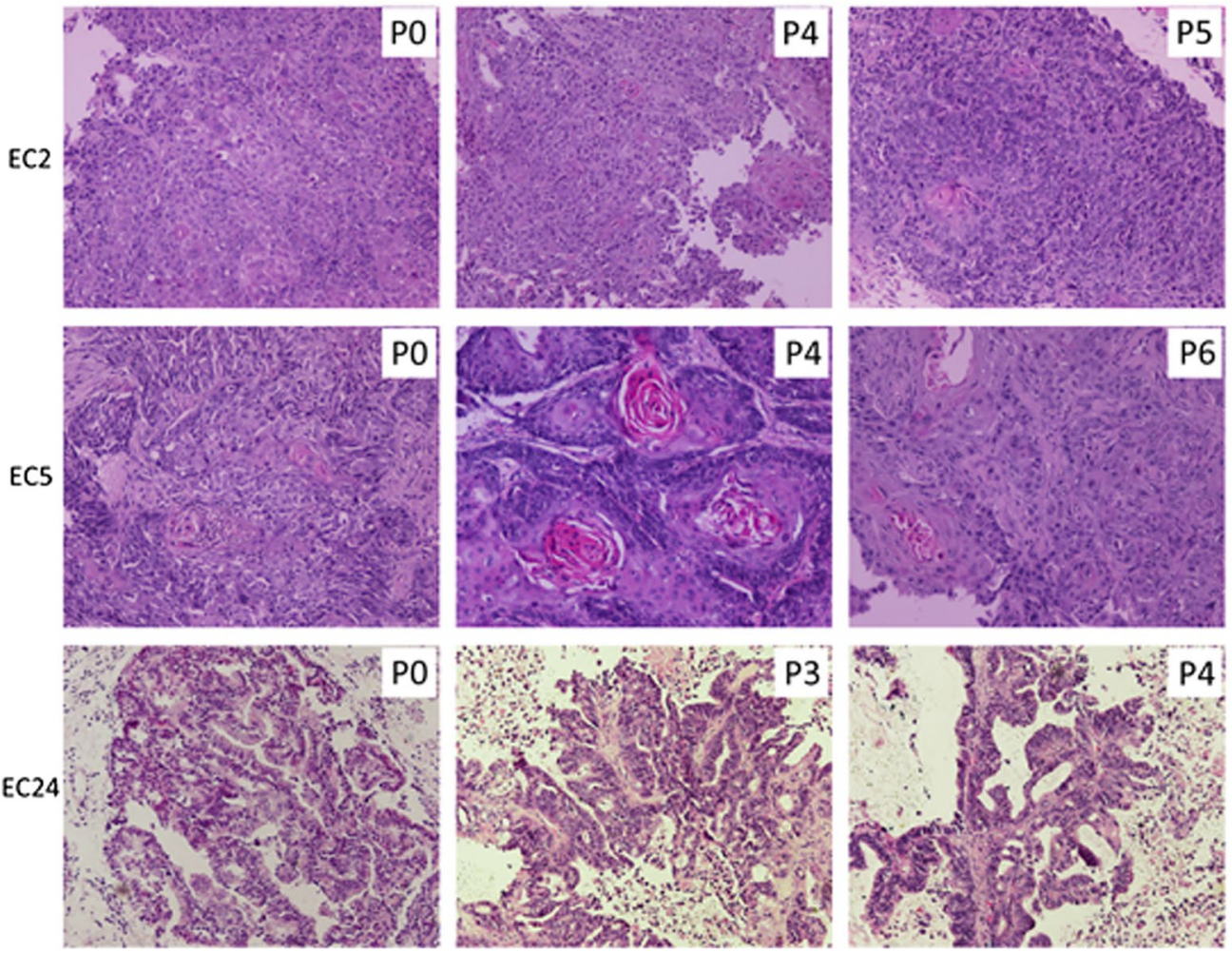

Fig. 1 Establishment and histological characterization of PDX models from esophageal cancer patients. a Diagram of establishment of a stable PDX bank. b Decreasing latency with increasing passage. Line and error bars represent mean \pm SD. $p$ values were calculated using an unpaired twotailed Student's $t$ test. ${ }^{*} p<0.05 ;{ }^{* *} p<0.01$. c Representative histology of paired patient-PDX tumors. Slides were stained with H\&E and images were captured at $\times 200$

tumors located in median (44\%) or lower (44\%) esophagus. Histopathological and treatment data was shown in Table 1.

\section{Histology of primary tumors and xenografts}

Pathological assessment with H\&E staining and analysis revealed consistent morphology and histology in xenografts compared with that of the corresponding primary tumor tissues (Fig. 1c; Additional file 1: Table S3). For case EC2, the PDXs kept poor differentiation from P1 to $\mathrm{P} 5$, which was in good accordance with the primary tumor. For case EC5, there was some variation in differentiation, with moderate differentiation in patient tumor and PDX of P1 to P6, except for P4, which was well differentiation (Fig. 1c). We then compared the histology type and differentiation of primary tumor and the two 
Table 1 Summary of characteristics of established PDX models for esophageal cancer (all male)

\begin{tabular}{|c|c|c|c|c|c|}
\hline Case no. & Age & Tumor location & Differentiation & Stage & Treatment status \\
\hline EC1 & 48 & Lower & Poor & IV & Progressive disease \\
\hline EC2 & 58 & Median & Poor & IV & Before treatment \\
\hline EC3 & 41 & Lower & Moderate & IV & Before treatment \\
\hline EC4 & 58 & Lower & Poor & IV & Before treatment \\
\hline EC5 & 51 & Median & Moderate & IV & Before treatment \\
\hline EC6 & 53 & Median & Poor & IV & Before treatment \\
\hline EC7 & 46 & Lower & Moderate & III & Stable disease \\
\hline EC8 & 49 & Lower & Poor & IV & Before treatment \\
\hline EC9 & 57 & Median & Moderate & III & Before treatment \\
\hline EC10 & 54 & Median & Poor & IV & Before treatment \\
\hline EC11 & 66 & Lower & Poor & IV & Before treatment \\
\hline EC12 & 49 & Lower & Moderate & IV & Before treatment \\
\hline EC13 & 68 & Lower & Moderate & IV & Before treatment \\
\hline EC14 & 55 & Upper & Moderate & IV & Before treatment \\
\hline EC15 & 63 & NA & Moderate & NA & NA \\
\hline EC16 & 62 & Median & Poor & NA & NA \\
\hline EC17 & 69 & Upper & Moderate & IV & Before treatment \\
\hline EC18 & 51 & Median & Moderate & III & Before treatment \\
\hline EC19 & 56 & Median & Moderate & IV & Before treatment \\
\hline EC20 & 79 & Median & Moderate & IV & Before treatment \\
\hline EC21 & 66 & Lower & Moderate & IV & Stable disease \\
\hline EC22 & 53 & Lower & Poor & IV & Before treatment \\
\hline EC23 & 59 & Median & Poor & IV & Before treatment \\
\hline $\mathrm{EC} 24^{\mathrm{a}}$ & 60 & Median & Moderate & IV & Before treatment \\
\hline$E C 25^{\mathrm{a}}$ & 68 & Lower & Moderate & NA & NA \\
\hline
\end{tabular}

All cases were of squamous carcinoma with the exception of those noted ${ }^{a}$ which were adenomas

NA non-available

consecutive generation of $\mathrm{P} 3, \mathrm{P} 4$, and found that all the cases keep the same histology, and most cases kept the differentiation consistent $(64 \%, 16 / 25)$, with some small changes in different passage (Additional file 1: Table S3).

\section{Sensitivity of PDX models to chemotherapy}

Five PDX models were treated with the clinically used chemotherapy agents, paclitaxel and cisplatin. Our data demonstrated that all of the PDX models had comparable therapeutic responses with that of the corresponding patients. The detailed data of the five PDX models and corresponding patients was shown in Fig. 2.

\section{Genomic characteristics of ESCC PDXs}

Using next generation sequencing (NGS) to molecularly profile xenografts, we identified 139 mutated genes from a total of 483 genes. Somatic mutations were variable (3-22; Fig. 3a, top). The observed mutation spectrum could be indicative of specific mutagenesis mechanisms occurring in ESCC. Among the 139 single-nucleotide variants, nucleotide transversion was over-represented, particularly C:G > T:A changes (Fig. 3a, bottom and right), which is consistent with ESCC patients in a previous study $[27,28]$.

Apart from the 139 genes with nonsynonymous single nucleotide variants (SNVs), 79 genes including insertions and deletions (InDels) in protein-coding regions were identified in the 23 PDXs. Thus, 191 non-silent somatic mutated genes were identified, with the range of 6-27 genes for each PDX (Fig. 3b, top). Of which, 96 genes were mutated in 2 or more samples. The top 10 mutated genes (frequency $\geq 7$ ) in the 23 PDX models, and the 10 frequently mutated genes from other reports [27-32] appear in Fig. 3b. KMT2D was the most frequently mutated gene, involving the 7 frameshift inDel, one nonframeshift inDel and 7 missense mutation followed by EP300, PIK3CA, ALK and ERBB2. Frequencies of somatic mutations for each gene appear in Fig. $3 \mathrm{~b}$ (right).

To interrogate the copy number alteration, 311 genes with copy number alterations (CNAs) were identified $($ median $=3.96$, range $2.8-39.18$ ). According to 

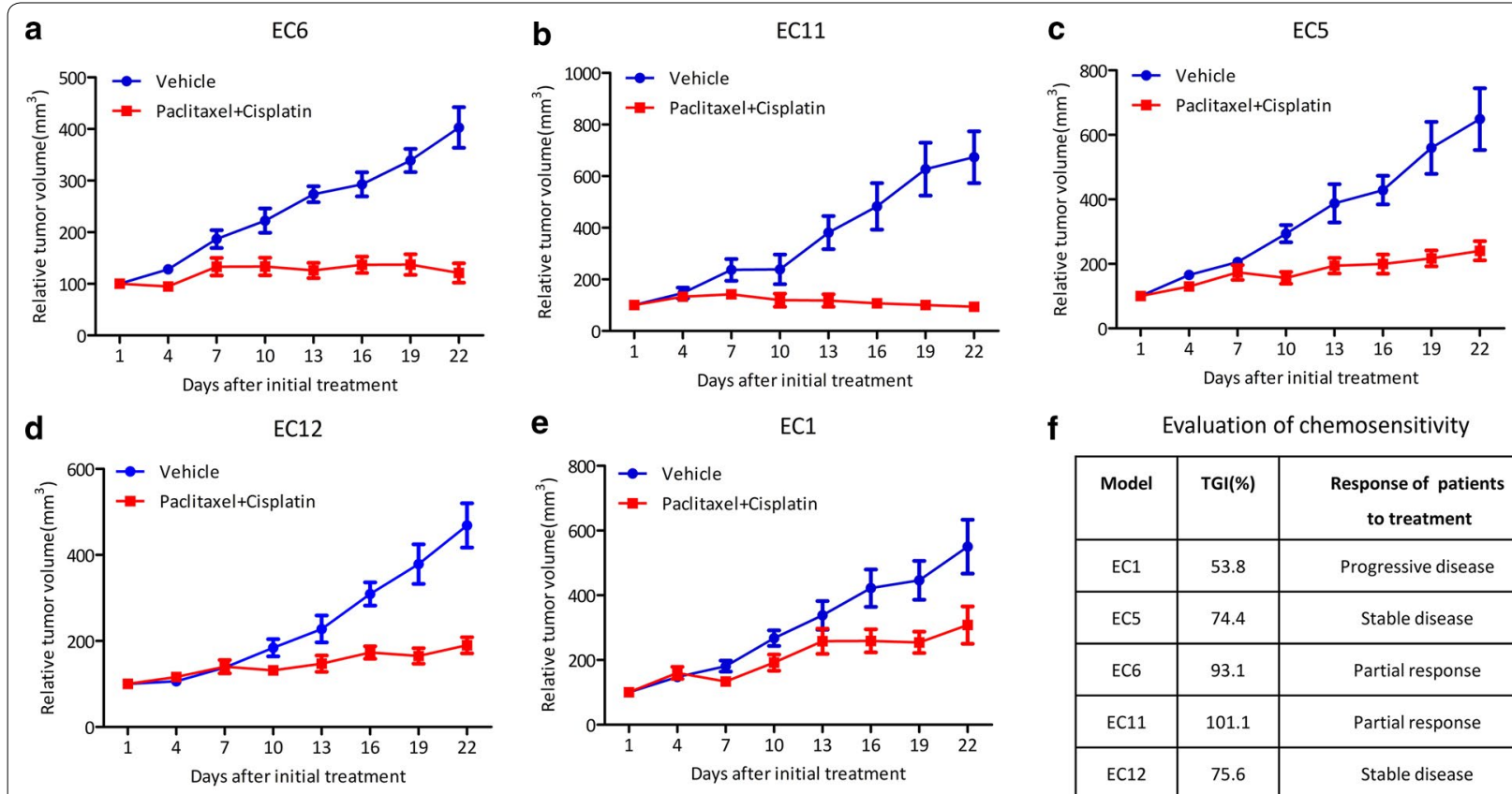

f Evaluation of chemosensitivity
\begin{tabular}{|c|c|c|}
\hline Model & TGI(\%) & $\begin{array}{c}\text { Response of patients } \\
\text { to treatment }\end{array}$ \\
\hline EC1 & 53.8 & Progressive disease \\
\hline EC5 & 74.4 & Stable disease \\
\hline EC6 & 93.1 & Partial response \\
\hline EC11 & 101.1 & Partial response \\
\hline EC12 & 75.6 & Stable disease \\
\hline
\end{tabular}

Fig. 2 Therapeutic response of PDX models and corresponding patients. Five PDX models of ESCC were evaluated for therapeutic responses compared to corresponding patients. Better response was seen in PDX models with tumor tissue from patients with partial responses (a, b) compared to those with stable disease $(\mathbf{c}, \mathbf{d})$. Case EC1 (patient with progressive disease) had a minimum response $(\mathbf{e})$. TGl and response for corresponding patients are given (f)

previous reports, genes with CNAs $\geq 5$ are considered amplified [33, 34]. Finally, 63 genes were identified. And, we found 14 genes that were amplified in two or more PDX models (Fig. 3c). FGF3 (13/23), FGF4 (13/23), FGF19 (12/23). CCND1 (12/23) were the most frequently amplified genes and all had an amplification peak spanning 11q13.3. Additional peaks involving important cancer genes such as SOX2, MYC, VEGFA and CDK6 were also found. To demonstrate the amplification, we detected the expression of FGF3, FGF4, FGF19 using quantitative real-time PCR as reported papers [35]. All the samples were classified into two groups (CNA $\geq 5$ and $\mathrm{CNA}<5$ ) according to the CNA tested by NGS, and CNAs $\geq 5$ were considered amplified as it was mentioned above. As was shown in Additional file 2: Figure S1, in agreement with the CNA analysis, the relative mRNA expression of FGF3 (Additional file 2: Figure S1a, $\mathrm{p}<0.001$ ), FGF4 (Additional file 2: Figure S1b, $\mathrm{p}<0.001$ ) and FGF19 (Additional file 2: Figure S1c, $\mathrm{p}<0.05$ ) was enhanced in amplification group $(\mathrm{CNA} \geq 5)$. The results was also in accordance with the previous studies [36,37], indicating that most of the highly amplified genes showed elevated expression, and the CNAs provided a useful reference for amplification analysis.

Abnormal genes with SNV, InDel or amplifications were analyzed with DAVID Bioinformatics Resources
6.7, and significantly altered pathways $(\mathrm{p}<0.05)$ were enriched and presented (Fig. 4a). The ErbB signaling pathway was the most significantly altered one, followed by the MAPK signaling pathway. Other pathways and abnormal genes appear in Fig. 4b. Most targets and pathways have been reported as candidate targets in carcinomas [7, 38-42]. These results suggested that the PDX models we established may be used for potential target selection and mechanism research.

\section{Discussion}

As an aggressive disease, esophageal cancer is known for limited therapeutic options. So models that enable functional studies translate into the clinic are invaluable. In this study, we have demonstrated that PDX model of esophageal cancer could be derived from very small biopsy specimens. And the histopathological features and chemosensitivity of PDXs were in great accordance with the corresponding primary tumors of patients.

However, the engraftment rate was only $13.3 \%$, which was lower than $34.1 \%$ of gastric cancer established using biopsied tissues in our previous reports, and that $34-54 \%$ of esophageal cancer in other studies [17, 20, 21, 43]. The difference may result from the following aspects: first, the type of disease. In spite of being gastrointestinal malignant, esophageal cancer is quite different with gastric cancer in the original sites of disease, pathogenesis, 


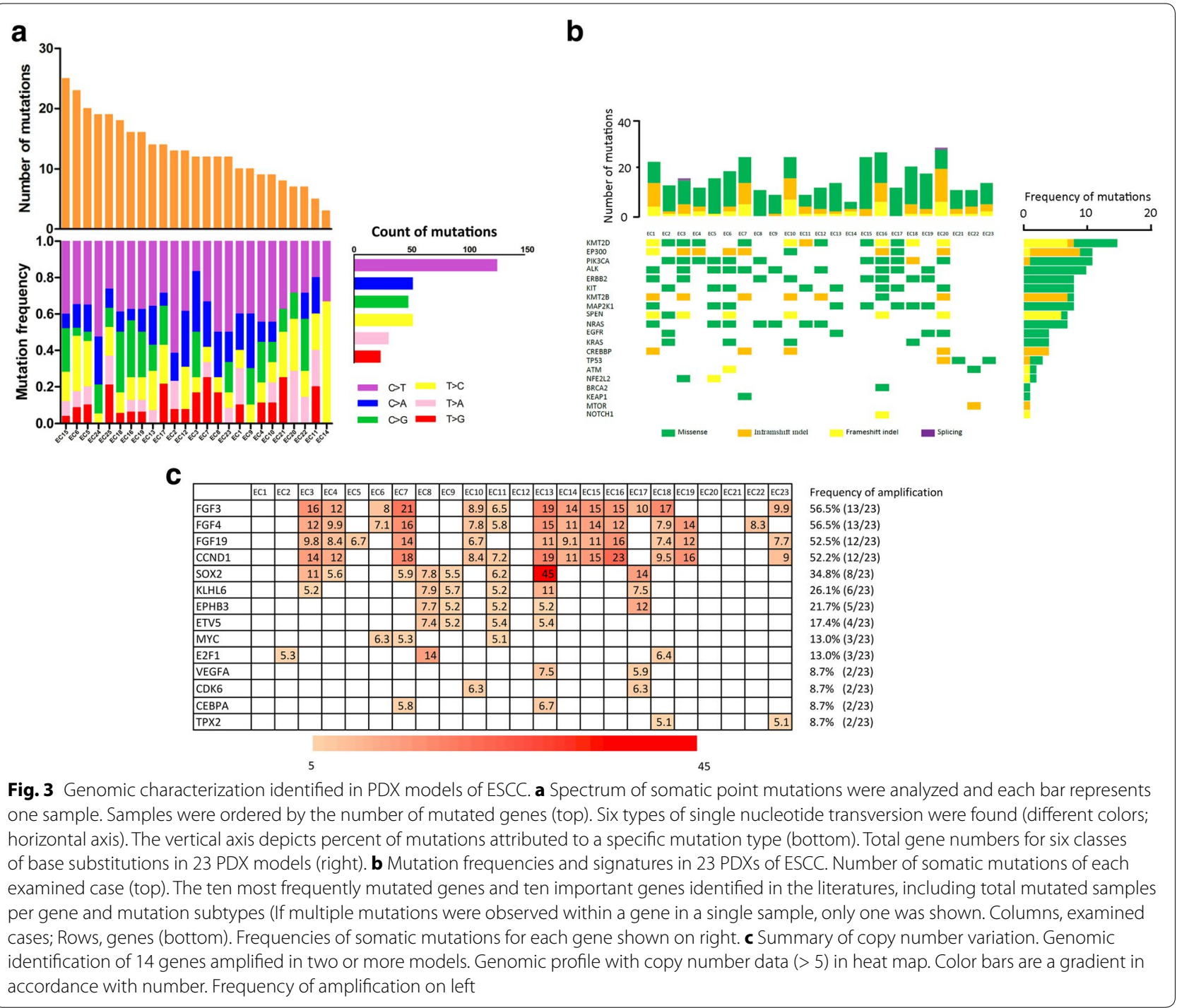

genetic profile and so on [44-47], which might lead to variant engraftment rate. Apart from this, the amount of primary tumor tissue might be an important factor influencing the rate of tumor formation. As it is known, it is not easy to get esophageal biopsy specimens for the special and thin anatomical structure. For the limited biopsy tissue, only one site of a mouse can be engrafted for each case. However, the tumor tissues from surgery are always sufficient to be implanted in multiple sites, which may increase the engraftment rate accordingly. Actually, most patients with esophageal cancer are diagnosed in advanced stage, and loss the opportunity of eradicative resection, so biopsy is the only way to establish PDX models. Besides, the paired biopsies before and after chemotherapy could help us to explore the drug-resistance mechanism in the PDX models.
Using our panel of xenograft models, we demonstrated that the model histology correlate well with the primary patient and remained similar between passages. However, some samples had small morphological variations with respect to differentiation perhaps due to the inherent heterogeneity of ESCC and the variability in sampling. ESCC is a type of carcinoma with high intratumoral heterogeneity [30]. Because all PDX models in our study were established with biopsied tissue, only a small fraction of primary tumor can be obtained for implantation, which may different from the specimen for diagnosis. That may explain partially the discrepant differentiation between PDX model and primary tissue. Also, difference might arise from the selection pressures during engraftment in different hosts [48]. Moreover, 5 xenograft models were treated with same chemotherapy agents to patients, and 


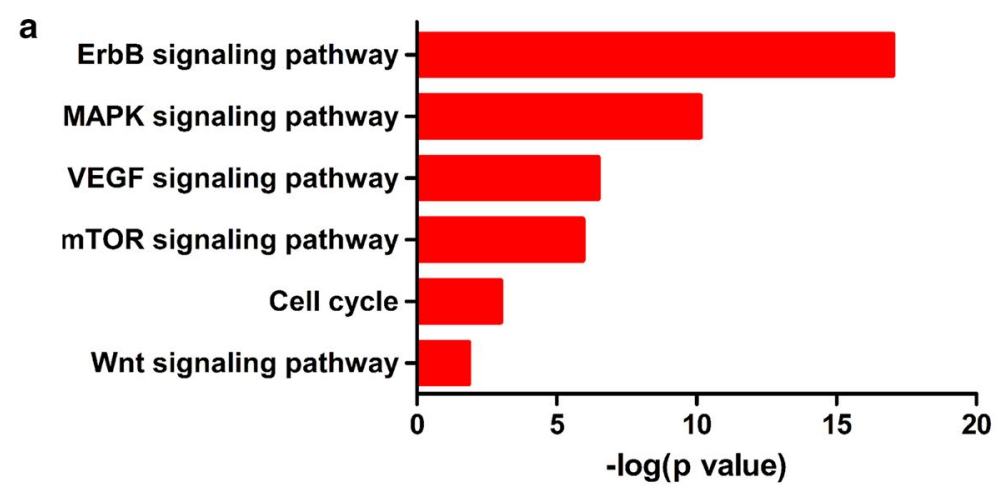

b
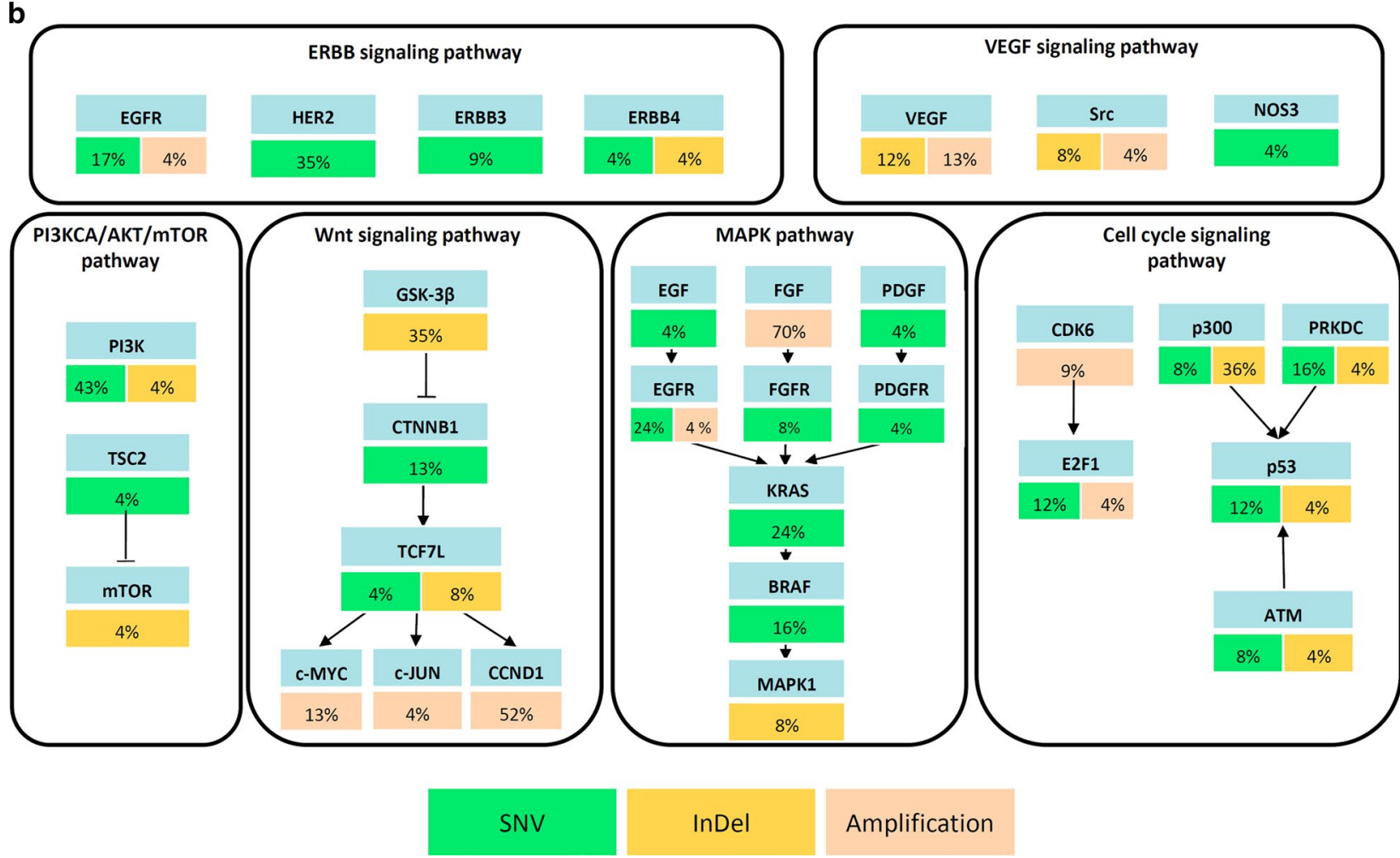

Fig. 4 Dysregulated pathways in PDX models of ESCC. a Significantly altered pathways $(p<0.05)$ were enriched and presented. $\mathbf{b}$ ESCC potential driver genes, mutation frequencies mapped and alteration frequencies shown as percent of all samples; genes identified as SNV are green; InDel are yellow; and amplified genes are pink. Excitatory (arrows) and inhibitory (black lines) interactions were obtained from the KEGG pathway database

response was in accordance with that of the patients. Our study showed very promising results that the drug sensitivity in PDX assays correlates with patient clinical response, which could provide a realistic model for drug sensitivity selection for ESCC patients.

Pharmacotherapy is the main treatment in advanced ESCC. However, the existing drugs showed little effect in prolonging the overall survival, so it is urgent to develop new agents for these patients. Current approaches of personalized medicine have been incorporating NGS technologies for wide genomic profiling of patient tumors to identify novel therapeutic targets. PDX models may provide a more feasible approach to develop novel agents, study the response to pharmacotherapies and explore predictive markers. The success of targeted therapies in stratifying treatment has underscored the importance of performing mechanistic and functional investigations on breast cancer, NSCLC, colon cancer, gastric cancer and so on $[49,50]$. In this study, we report extensive molecular characterization of the 23 ESCC PDX models using NGS technology. The results showed ErbB, MAPK, VEGF, mTOR, cell cycle, and Wnt signaling pathway were mostly frequent abnormal pathways, which were similar to previous reports from ESCC patients [35, 51-53]. 
However, there is still no effective targeted therapy for ESCC patients. So the labeled mice model corresponding to certain gene or pathway could be available for preclinical evaluation of targeted drug candidates, which will be useful for further application. For example, in our previous study, the anti-tumor effect of CDK4/6 inhibitor SHR6390 was demonstrated in ESCC [54]. And by analyzing the CDK6 expression in esophageal PDX model and transfecting esophageal cancer cell line with small interfering RNA, we found that the CDK6 expressions may be a useful marker to identify the patients who are more likely to benefit from treatment with SHR6390. Apart from it, somatic mutations in the tyrosine-kinase domain of EGFR were identified in 30-50\% non-small-cell lung cancers (NSCLCs) patients, among which the TKIs response rate increased to approximately $75 \%[55,56]$. But the EGFR mutations appear to be a rare studied field in ESCC. In our study, the disrupted ERBB pathway including mutation in ERBB2 (35\%), which can be used to explore the TKIs therapy in esophageal cancer. Besides, in a cohort of metastatic renal cell carcinoma patients, mutations in TSC2 were more common in patients who experienced clinical benefit from mTOR inhibitors than in those who progressed [57], indicating that the mutation of TSC2 would be a good prognosis indicator for mTOR inhibitors. But there is no related studies in esophageal cancer, so the PDX models with TSC2 mutation will provided a useful tool.

There are also limitations which should be emphasized. Firstly, the PDX model were established on the NOD/ SCID mice, which is lack of integrated immune system. This need to be careful when considering immunological therapies. However, the emerging humanized immune mice model can be provided as a promising model which will solve the problem in a great extent. PDX models can also be a live biobank to offer tumor tissue to establish a more advanced preclinical model which imitates the tumor and immune phenotype partially. Secondly, due to the small volume of the biopsy samples, we could not get the enough samples to validate the molecular characteristic between the PDX and corresponding primary tumor in patients. The establishment of ESCC PDX models in this study was being continued and the rapid improvement in quality, quantity, and cost of NGS will help with this.

\section{Conclusions}

We have shown that PDX models of ESCC can be generated from small biopsy samples and used for functional and genomic studies. It could provide a reliable model for developing new agents and exploring the predictive markers, and therefore provide evidence for individual therapy.

\section{Additional file}

Additional file 1: Table S1. The list of 483 cancer-associated genes. Table S2. Patient characteristics and transplantation rate. Table S3. Evaluation of histology and differentiation between primary patient tumor and the xenografts.

Additional file 2: Figure S1. Correlation between mRNA expression and CAN of FGF3 (a), FGF4 (b), and FGF19 (c) in esophageal PDX samples. The graph show mean values for two groups $(C N V \geq 5$ or $C N V<5)$. The mRNA expression of the gene of interest was expressed in relation to that of $\beta$-actin, used as a housekeeping gene. Error bars represent \pm S.E.M. (t-test).

\section{Abbreviations}

PDX: patient-derived xenografts; ESCC: esophageal squamous cell carcinoma; SNV: single nucleotide variant; InDel: insertion and deletion; CNA: copy number alteration; NGS: next generation sequencing.

\section{Authors' contributions}

$L S$ and JG conceived and designed the study. JZ and YL carried out most of the experimental work and contributed to manuscript writing. JW and ZL collected the tissues. ZC and WH performed the functional and pathway analysis. $\mathrm{ZL}, \mathrm{BD}$ and $\mathrm{YL}$ carried out and analyzed histology of primary tumors and xenografts. ZL proposed several experiments and contributed to manuscript writing. All authors read and approved the final manuscript.

\section{Author details}

${ }^{1}$ Department of Gastrointestinal Oncology, Key Laboratory of Carcinogenesis and Translational Research (Ministry of Education), Peking University Cancer Hospital \& Institute, Fu-Cheng Road 52, Hai-Dian District, Beijing 100142, China. ${ }^{2}$ Laboratory of Genetics, Key Laboratory of Carcinogenesis and Translational Research (Ministry of Education), Peking University Cancer Hospital \& Institute, Beijing 100142, China. ${ }^{3}$ Department of Pathology, Key Laboratory of Carcinogenesis and Translational Research (Ministry of Education), Peking University Cancer Hospital \& Institute, Beijing, China.

\section{Acknowledgements}

We thank LetPub (http://www.letpub.com) for its linguistic assistance during the preparation of this manuscript.

\section{Competing interests}

The authors declare that they have no competing interests.

\section{Availability of data and materials}

All data generated or analyzed during this study are included in this published article (and its additional files).

\section{Consent for publication \\ Not applicable.}

\section{Ethics approval and consent to participate}

The study was approved and supervised by the research ethics committee of Peking University Cancer Hospital \& Institute, Beijing, China (No. 2016KT81). All animal experiments were performed in accordance with the animal experimental guidelines of Peking University Cancer Hospital and followed internationally recognized ARRIVE (Animal Research: Reporting of In Vivo Experiments) guidelines. The study was conducted in accordance with the principles expressed in the Declaration of Helsinki.

\section{Funding}

This work was supported by Beijing Municipal Administration of Hospital Program (QML20151002), Beijing Municipal Senior Technical Training Plan in Health System (2015-3-073), and Beijing Municipal Science and Technology (Z141100002114046). 


\section{Publisher's Note}

Springer Nature remains neutral with regard to jurisdictional claims in published maps and institutional affiliations.

Received: 5 October 2017 Accepted: 5 January 2018

Published online: 25 January 2018

\section{References}

1. Chen W, Zheng R, Baade PD, Zhang S, Zeng H, Bray F, Jemal A, Yu XQ, He J. Cancer statistics in China, 2015. CA Cancer J Clin. 2016;66(2):115-32.

2. Arnold M, Soerjomataram I, Ferlay J, Forman D. Global incidence of oesophageal cancer by histological subtype in 2012. Gut. 2015;64(3):381-7.

3. Malhotra GK, Yanala U, Ravipati A, Follet M, Vijayakumar M, Are C. Global trends in esophageal cancer. J Surg Oncol. 2017;115(5):564-79.

4. De Angelis R, Sant M, Coleman MP, Francisci S, Baili P, Pierannunzio D, Trama A, Visser O, Brenner H, Ardanaz E, et al. Cancer survival in Europe 1999-2007 by country and age: results of EUROCARE-5-a populationbased study. Lancet Oncol. 2014;15(1):23-34.

5. Siegel R, Ma J, Zou Z, Jemal A. Cancer statistics, 2014. CA Cancer J Clin. 2014;64(1):9-29.

6. Sivanand S, Pena-Llopis S, Zhao H, Kucejova B, Spence P, Pavia-Jimenez A, Yamasaki T, McBride DJ, Gillen J, Wolff NC, et al. A validated tumorgraft model reveals activity of dovitinib against renal cell carcinoma. Sci Trans Med. 2012;4(137):175

7. Belkhiri A, El-Rifai W. Advances in targeted therapies and new promising targets in esophageal cancer. Oncotarget. 2015;6(3):1348-58.

8. Liu Y, Xiong Z, Beasley A, D'Amico T, Chen XL. Personalized and targeted therapy of esophageal squamous cell carcinoma: an update. Ann NY Acad Sci. 2016;1381(1):66-73.

9. Xiong Z, He J, Chen XL. New strategies in esophageal carcinoma: promises and problems. J Thorac Dis. 2016;8(11):E1501-4.

10. Sathyanarayanan V, Neelapu SS. Cancer immunotherapy: strategies for personalization and combinatorial approaches. Mol Oncol. 2015;9(10):2043-53.

11. Tomei S, Wang E, Delogu LG, Marincola FM, Bedognetti D. Non-BRAF-targeted therapy, immunotherapy, and combination therapy for melanoma. Exp Opin Biol Ther. 2014;14(5):663-86.

12. Parchment RE, Voth AR, Doroshow JH, Berzofsky JA. Immuno-pharmacodynamics for evaluating mechanism of action and developing immunotherapy combinations. Semin Oncol. 2016:43(4):501-13.

13. Gao H, Korn JM, Ferretti S, Monahan JE, Wang Y, Singh M, Zhang C, Schnell C, Yang G, Zhang Y, et al. High-throughput screening using patientderived tumor xenografts to predict clinical trial drug response. Nat Med. 2015;21(11):1318-25.

14. Hidalgo M, Amant F, Biankin AV, Budinska E, Byrne AT, Caldas C, Clarke RB, de Jong S, Jonkers J, Maelandsmo GM, et al. Patient-derived xenograft models: an emerging platform for translational cancer research. Cancer Discov. 2014;4(9):998-1013.

15. Cancer Genome Atlas Research N. Comprehensive genomic characterization of squamous cell lung cancers. Nature. 2012;489(7417):519-25.

16. Zhu Y, Tian T, Li Z, Tang Z, Wang L, Wu J, Li Y, Dong B, Li Y, Li N, et al. Establishment and characterization of patient-derived tumor xenograft using gastroscopic biopsies in gastric cancer. Sci Rep. 2015;5:8542.

17. Jiang Y, Wu Q, Yang X, Zhao J, Jin Y, Li K, Ma Y, Chen X, Tian F, Zhao S, et al. A method for establishing a patient-derived xenograft model to explore new therapeutic strategies for esophageal squamous cell carcinoma. Oncol Rep. 2016:35(2):785-92.

18. Read M, Liu D, Duong CP, Cullinane C, Murray WK, Fennell CM, Shortt J, Westerman D, Burton P, Clemons NJ, et al. Intramuscular transplantation improves engraftment rates for esophageal patient-derived tumor xenografts. Ann Surg Oncol. 2016;23(1):305-11.

19. Sun S, Zhang Z. Patient-derived xenograft platform of OSCC: a renewable human bio-bank for preclinical cancer research and a new co-clinical model for treatment optimization. Front Med. 2016:10(1):104-10.

20. Wu X, Zhang J, Zhen R, Lv J, Zheng L, Su X, Zhu G, Gavine PR, Xu S, Lu $S$, et al. Trastuzumab anti-tumor efficacy in patient-derived esophageal squamous cell carcinoma xenograft (PDECX) mouse models. J Transl Med. 2012;10:180

21. Zhang J, Jiang D, Li X, Lv J, Xie L, Zheng L, Gavine PR, Hu Q, Shi Y, Tan L, et al. Establishment and characterization of esophageal squamous cell carcinoma patient-derived xenograft mouse models for preclinical drug discovery. Lab Invest J Tech Methods Pathol. 2014;94(8):917-26.

22. Chang L, Guo F, Lv Y, Wang Y, Huo B, Wang L, Liu W. The inhibitory effects of Endostar combined with chemotherapy on human esophageal squamous cell carcinoma xenograft in mice. Mol Biol Rep. 2013;40(1):669-73.

23. Chang L, Huo B, Lv Y, Wang Y, Liu W. Ginsenoside Rg3 enhances the inhibitory effects of chemotherapy on esophageal squamous cell carcinoma in mice. Mol Clin Oncol. 2014;2(6):1043-6.

24. Sun J, Deng L, Duan Y, Chen F, Wang X, Li D, Chen Z. Inhibitory effect of endostatin combined with paclitaxel-cisplatin on breast cancer in xenograft-bearing mice. Exp Ther Med. 2012;3(2):159-64.

25. Gao J, Wang H, Zang W, Li B, Rao G, Li L, Yu Y, Li Z, Dong B, Lu Z, et al. Circulating tumor DNA functions as an alternative for tissue to overcome tumor heterogeneity in advanced gastric cancer. Cancer Sci. 2017;108:1881-7

26. Yoon S, Xuan Z, Makarov V, Ye K, Sebat J. Sensitive and accurate detection of copy number variants using read depth of coverage. Genome Res. 2009:19(9):1586-92.

27. Zhang L, Zhou Y, Cheng C, Cui H, Cheng L, Kong P, Wang J, Li Y, Chen W, Song B, et al. Genomic analyses reveal mutational signatures and frequently altered genes in esophageal squamous cell carcinoma. Am J Hum Genet. 2015;96(4):597-611.

28. Song Y, Li L, Ou Y, Gao Z, Li E, Li X, Zhang W, Wang J, Xu L, Zhou Y, et al. Identification of genomic alterations in oesophageal squamous cell cancer. Nature. 2014:509(7498):91-5.

29. Cheng C, Zhou Y, Li H, Xiong T, Li S, Bi Y, Kong P, Wang F, Cui H, Li Y, et al. Whole-genome sequencing reveals diverse models of structural variations in esophageal squamous cell carcinoma. Am J Hum Genet. 2016:98(2):256-74.

30. Hao JJ, Lin DC, Dinh HQ, Mayakonda A, Jiang YY, Chang C, Jiang Y, Lu CC, Shi ZZ, Xu X, et al. Spatial intratumoral heterogeneity and temporal clonal evolution in esophageal squamous cell carcinoma. Nat Genet. 2016;48(12):1500-7.

31. Lin DC, Hao JJ, Nagata Y, Xu L, Shang L, Meng X, Sato Y, Okuno Y, Varela AM, Ding LW, et al. Genomic and molecular characterization of esophageal squamous cell carcinoma. Nat Genet. 2014:46(5):467-73.

32. Sasaki Y, Tamura M, Koyama R, Nakagaki T, Adachi Y, Tokino T. Genomic characterization of esophageal squamous cell carcinoma: insights from next-generation sequencing. World J Gastroenterol. 2016;22(7):2284-93.

33. Pritchard CC, Salipante SJ, Koehler K, Smith C, Scroggins S, Wood B, WU D, Lee MK, Dintzis S, Adey A, et al. Validation and implementation of targeted capture and sequencing for the detection of actionable mutation, copy number variation, and gene rearrangement in clinical cancer specimens. J Mol Diagn JMD. 2014;16(1):56-67.

34. Ohshima K, Hatakeyama K, Nagashima T, Watanabe Y, Kanto K, Doi Y, Ide T, Shimoda Y, Tanabe T, Ohnami S, et al. Integrated analysis of gene expression and copy number identified potential cancer driver genes with amplification-dependent overexpression in 1454 solid tumors. Sci Rep. 2017:7(1):641.

35. Chin SF, Teschendorff AE, Marioni JC, Wang Y, Barbosa-Morais NL, Thorne NP, Costa JL, Pinder SE, van de Wiel MA, Green AR, et al. High-resolution aCGH and expression profiling identifies a novel genomic subtype of ER negative breast cancer. Genome Biol. 2007;8(10):R215.

36. Pollack JR, Sorlie T, Perou CM, Rees CA, Jeffrey SS, Lonning PE, Tibshirani $\mathrm{R}$, Botstein D, Borresen-Dale AL, Brown PO. Microarray analysis reveals a major direct role of DNA copy number alteration in the transcriptional program of human breast tumors. Proc Natl Acad Sci USA. 2002;99(20):12963-8.

37. Hodgson JG, Yeh RF, Ray A, Wang NJ, Smirnov I, Yu M, Hariono S, Silber J, Feiler HS, Gray JW, et al. Comparative analyses of gene copy number and mRNA expression in glioblastoma multiforme tumors and xenografts. Neurooncology. 2009;11(5):477-87.

38. Saxton RA, Sabatini DM. mTOR signaling in growth, metabolism, and disease. Cell. 2017;168(6):960-76.

39. Madhusudan S, Hickson ID. DNA repair inhibition: a selective tumour targeting strategy. Trends Mol Med. 2005;11(11):503-11. 
40. Waldner MJ, Neurath MF. Targeting the VEGF signaling pathway in cancer therapy. Exp Opin Ther Targets. 2012;16(1):5-13.

41. Fang JY, Richardson BC. The MAPK signalling pathways and colorectal cancer. Lancet Oncol. 2005;6(5):322-7.

42. Baselga J, Swain SM. Novel anticancer targets: revisiting ERBB2 and discovering ERBB3. Nat Rev Cancer. 2009;9(7):463-75.

43. Dodbiba L, Teichman J, Fleet A, Thai H, Starmans MH, Navab R, Chen Z Girgis H, Eng L, Espin-Garcia O, et al. Appropriateness of using patientderived xenograft models for pharmacologic evaluation of novel therapies for esophageal/gastro-esophageal junction cancers. PLoS ONE. 2015:10(3):e0121872.

44. Denlinger CE, Thompson RK. Molecular basis of esophageal cancer development and progression. Surg Clin N Am. 2012;92(5):1089-103.

45. Zhang Y. Epidemiology of esophageal cancer. World J Gastroenterol. 2013;19(34):5598-606

46. Hamashima C. Current issues and future perspectives of gastric cancer screening. World J Gastroenterol. 2014;20(38):13767-74.

47. Figueiredo C, Camargo MC, Leite M, Fuentes-Panana EM, Rabkin CS, Machado JC. Pathogenesis of gastric cancer: genetics and molecular classification. Curr Top Microbiol Immunol. 2017;400:277-304.

48. Aparicio S, Hidalgo M, Kung AL. Examining the utility of patient-derived xenograft mouse models. Nat Rev Cancer. 2015;15(5):311-6.

49. Jones KL, Buzdar AU. Evolving novel anti-HER2 strategies. Lancet Oncol. 2009;10(12):1179-87.

50. Dancey JE, Bedard PL, Onetto N, Hudson TJ. The genetic basis for cancer treatment decisions. Cell. 2012;148(3):409-20.

51. Wu C, Hu Z, He Z, Jia W, Wang F, Zhou Y, Liu Z, Zhan Q, Liu Y, Yu D, et al. Genome-wide association study identifies three new susceptibility loci for esophageal squamous-cell carcinoma in Chinese populations. Nat Genet. 2011;43(7):679-84.

52. Liu W, Snell JM, Jeck WR, Hoadley KA, Wilkerson MD, Parker JS, Patel N, Mlombe YB, Mulima G, Liomba NG, et al. Subtyping sub-Saharan esophageal squamous cell carcinoma by comprehensive molecular analysis. JCl Insight. 2016;1 (16):e88755.

53. Hu N, Kadota M, Liu H, Abnet CC, Su H, Wu H, Freedman ND, Yang $\mathrm{HH}$, Wang C, Yan C, et al. Genomic landscape of somatic alterations in esophageal squamous cell carcinoma and gastric cancer. Can Res. 2016;76(7):1714-23.

54. Wang J, Li Q, Yuan J, Wang J, Chen Z, Liu Z, Li Z, Lai Y, Gao J, Shen L. CDK4/6 inhibitor-SHR6390 exerts potent antitumor activity in esophageal squamous cell carcinoma by inhibiting phosphorylated $\mathrm{Rb}$ and inducing G1 cell cycle arrest. J Transl Med. 2017;15(1):127.

55. Suzuki R, Hasegawa Y, Baba K, Saka H, Saito H, Taniguchi H, Yamamoto M, Matsumoto S, Kato K, Oishi T, et al. A phase II study of single-agent gefitinib as first-line therapy in patients with stage IV non-small-cell lung cancer. Br J Cancer. 2006;94(11):1599-603.

56. Inoue A, Suzuki T, Fukuhara T, Maemondo M, Kimura Y, Morikawa N, Watanabe H, Saijo Y, Nukiwa T. Prospective phase II study of gefitinib for chemotherapy-naive patients with advanced non-small-cell lung cancer with epidermal growth factor receptor gene mutations. J Clin Oncol Off J Am Soc Clin Oncol. 2006;24(21):3340-6.

57. Kwiatkowski DJ, Choueiri TK, Fay AP, Rini BI, Thorner AR, de Velasco G, Tyburczy ME, Hamieh L, Albiges L, Agarwal N, et al. Mutations in TSC1, TSC2, and MTOR are associated with response to rapalogs in patients with metastatic renal cell carcinoma. Clin Cancer Res Off J Am Assoc Cancer Res. 2016;22(10):2445-52.

\section{Submit your next manuscript to BioMed Central and we will help you at every step:}

- We accept pre-submission inquiries

- Our selector tool helps you to find the most relevant journal

- We provide round the clock customer support

- Convenient online submission

- Thorough peer review

- Inclusion in PubMed and all major indexing services

- Maximum visibility for your research

Submit your manuscript at www.biomedcentral.com/submit 